\title{
RF FREQUENCY SHIFT DURING BEAM STORAGE IN THE SLC DAMPING RINGS *
}

\author{
R. Akre, F. -J. Decker, M.G. Minty \\ Stanford Linear Accelerator Center (SLAC), Stanford, CA USA
}

\section{Abstract}

A method to reduce the horizontal damping time and equilibrium emittance of the SLC Damping Rings required changing the RF frequency during the beam storage time. The changed frequency causes the beam to pass off center through the quadrupoles effectively stretching the ring. The timing and phasing of the ring is required to be locked to the accelerator for both injection and extraction. The requirement was to change the RF frequency by up to $100 \mathrm{kHz}$ after injection transients were damped. Before extraction from the ring, the bunch had to be in the correct bucket and phase locked to the linac. It was necessary that the frequency shift not interfere with the operation of several feedback loops and that any stimulated bunch oscillations be damped to less than $0.1^{\circ}$ at $714 \mathrm{MHz}$ before extraction, less than $200 \mu \mathrm{s}$ after returning to the nominal frequency of $714.000 \mathrm{MHz}$. Several methods were evaluated to perform the task. The modifications made to the ring's RF system and operating parameters to accomplish the intrastore frequency shift are described.

\section{QUEST FOR A METHODE TO CHANGE FREQUENCY}

During past experiments unlocking of the ring oscillator, changing to a different frequency for a period of time, and then relocking had been done to shift timing of the extracted beam. Frequency shifts on the order of several kilohertz had been successfully accomplished by unlocking the rings phase locked oscillator from is reference source, however locking would take place several milliseconds before extraction. The current requirement of changing the frequency back to the correct phase and relocking to maintain an extraction jitter of less than $0.1^{\circ}$ RMS within $200 \mu$ s did not seem possible using existing hardware.

The ring oscillator used was not able to stabilize after a $100 \mathrm{kHz}$ frequency shift in time for extraction. Attempts were made to inject a $100 \mathrm{kHz}$ error signal into the feedback loop of the phase-locked oscillator. This method seemed promising although side band noise levels were higher than desired. Use of the ring oscillator to change frequency was set aside as a new method was tested.

* Work supported by the U.S. Department of Energy, contract DEAC03-76SF00515
Rather than change the frequency of the reference RF to the ring, a phase rotation could be used to cause a frequency change. Adding a phase rotation to a reference frequency results in a new frequency, above or below the reference depending on the direction of the phase rotation. The constant phase change shown in Figure 1 is equal to $360^{\circ}$ per 10 cycles of RF. This causes the frequency to increase by $10 \%$.

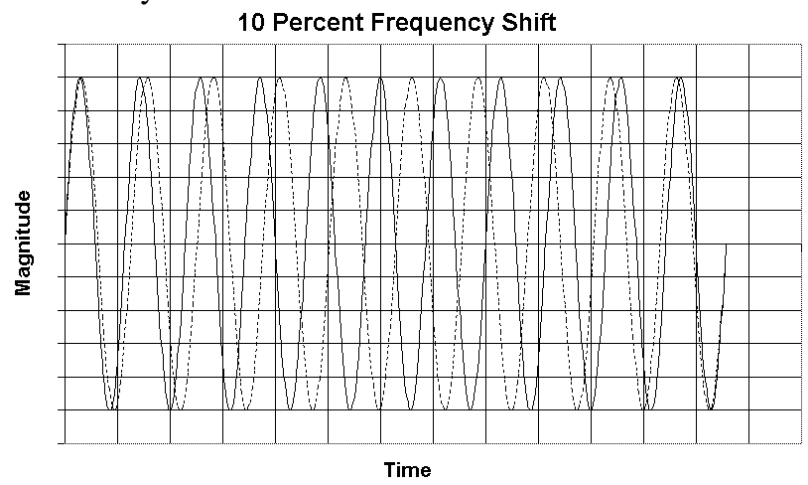

Figure 1. Example of frequency shift due to addition of constant phase change.

An In-phase and Quadrature-phase, I\&Q, modulator was built using two RF mixers, two power splitters, two 20dB attenuators, and an amplifier (see Fig. 2). An RF mixer has the property that when the local oscillator, LO, port is biased with RF, the RF port output amplitude is proportional to the intermediate frequency, IF, port input amplitude. As the IF input changes polarity, the RF output changes phase by $180^{\circ}$.

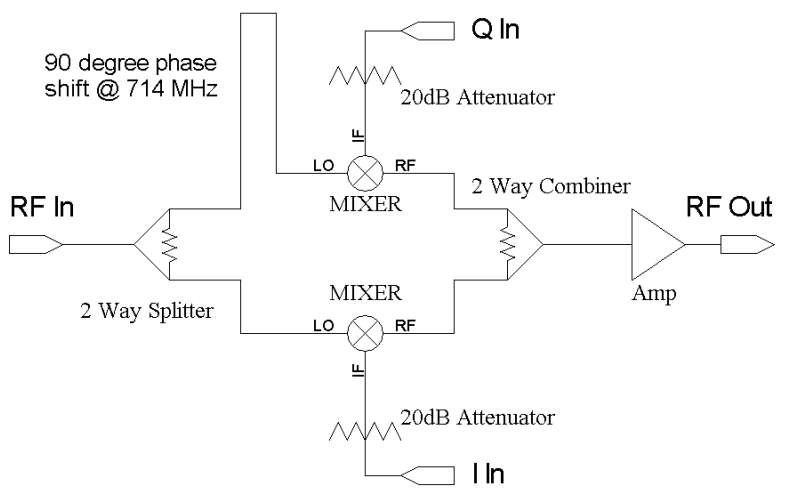

Figure 2. I \& Q Modulator.

The power into an I\&Q modulator is split with one of the RF paths being $90^{\circ}$ longer than the other. The RF is then used to drive the LO ports of the two mixers. The RF output ports of the two mixers are combined, amplified and then sent out. A $20 \mathrm{~dB}$ attenuator was placed on the 
IF port of each mixer to be able to drive the mixer with a voltage source. With a linear combination of the two orthogonal vectors any vector can be created. The voltage to the I \& Q inputs were adjusted to give a constant RF amplitude output for different RF output phases. The I \& $\mathrm{Q}$ voltages were then recorded (see Fig. 3).

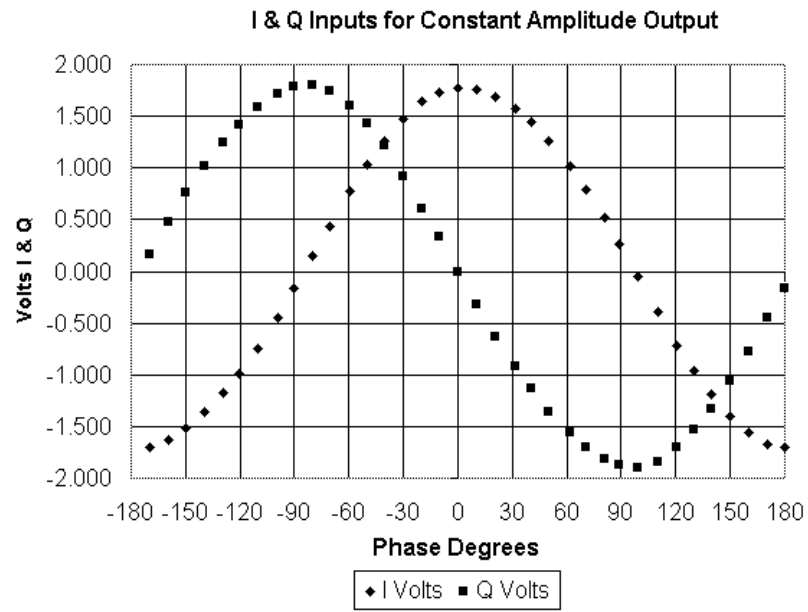

Figure 3. Calibration Data of the I \& Q Modulator.

Two arbitrary function generators were programmed to drive the $\mathrm{I}$ and $\mathrm{Q}$ mixers. Software was written to create waveforms for the I and Q arbitrary function generators. The function generators were triggered using the rings timing system. The predetermined waveform would run for the specified amount of time.

\section{DAMPING RING FEEDBACKS}

The system was installed in the damping ring as shown in Figure 5. There are two phase references for the damping ring. The RF, which drives the ring, is divided down from the $2856 \mathrm{MHz}$ at the injector. This RF is used to drive the ring and set the injection phase. The second phase reference comes from sector 2 where the damping ring bunch is injected. During the store the bunch phase is locked to the $2856 \mathrm{MHz}$ from sector 2 for extraction.

\subsection{Cavity Phase Angle}

The cavity phase measurement measures the phase between the forward RF into the cavity and the RF in the cavity. The measurement phase error is small since the difference in cable length between the forward RF coupler and the cavity probe to the phase detector is small. With a frequency change of $100 \mathrm{kHz}$ at $714 \mathrm{MHz}(140 \mathrm{ppm})$ a 40 ns difference in cable length would cause a $1.5^{\circ}$ error.

A frequency change for a resonant cavity is similar to a tuning angle change for the cavity. The cavity being run off frequency would require more RF power as the frequency changed. From Figure 4 an $80 \mathrm{kHz}$ offset in frequency would cause a $7 \mathrm{~dB}$ drop in cavity power and a $-60^{\circ}$ change in phase angle. Near resonance the cavity phase angle changes about $1.2^{\circ}$ per $\mathrm{kHz}$. In order to minimize the amount of power required the tuning angle should be set to $+36^{\circ}$ at $714 \mathrm{MHz}$. A frequency shift of $+80 \mathrm{kHz}$ would then change the tuning angle to $-36^{\circ}$, requiring the same klystron power for the same gap voltage. The RF phase measurement for the loop is sampled-and-held during a time after the beam has stabilized and before any change in frequency.
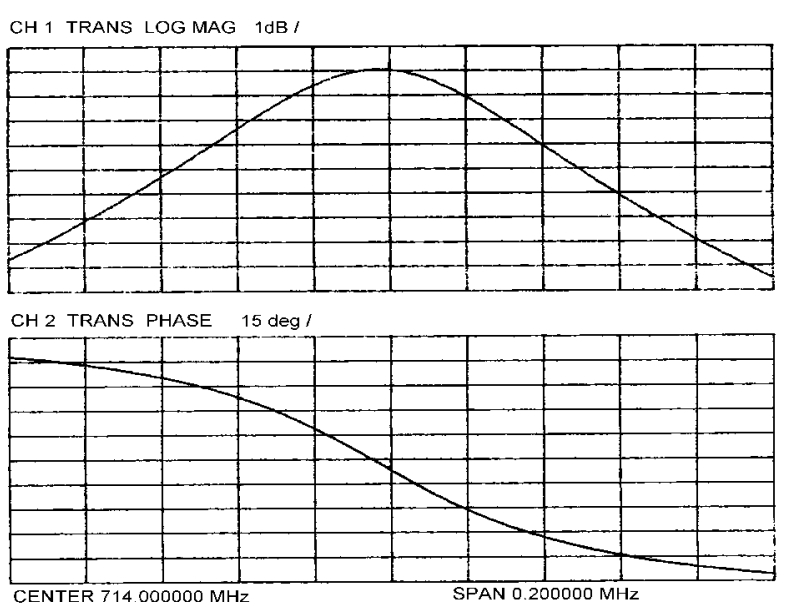

Figure 4. Cavity 591 Probe RF - Cavity Forward RF.

\subsection{Klystron Phase Loop}

The klystron phase loop measures the phase of the RF coming into the ring and compares it with the amplitude sum of the cavities. The loop is about 900ns in length. A $140 \mathrm{ppm}$ frequency change in the ring would cause a phase reading error of $33^{\circ}$. The loop is a slow loop which purpose is to compensate for phase drifts caused by temperature change and maintain the correct injection phase. During operation of the frequency change the feedback was turned off.

\subsection{Direct Feedback Loop}

The direct feedback loop mixes the vector sum of the cavities with the input drive RF to the klystron to stabilize transients. The length of the loop is about 350ns and in order to be stable, the loop must stay within a limited phase range around $180^{\circ}$ (negative feedback). A frequency change of $140 \mathrm{ppm}$ will cause a phase change in the feedback loop of $13^{\circ}$. The cavity phase change will dominate this value. Beam intensity changes in the cavities will also change the feedback phase. The gain of the loop was set low enough to allow all combinations of beam/no beam and frequency shift/no frequency shift to be stable.

\subsection{Extraction Phase Feedback}

Every $360^{\circ}$ rotation of the phase causes the bunch to shift a bucket with respect to where it would have been without the phase rotation. In order for the bunch to be extracted the same as if there would be no phase rotation it must precess the ring an integer number of turns. This places the bunch in the exact place it would have been if there 
were no frequency shift. The bunch passes through the extraction kicker at the exact same time it would have with no frequency shift and is injected into the linac in the same bucket. The phase ramp, which is required to compensate for the difference between the injection and extraction phase, is accomplished by stopping the phase rotation at the correct extraction phase. In order to maintain collisions it is necessary to keep track of the bucket the bunches are in and inject them into the correct accelerator bucket.

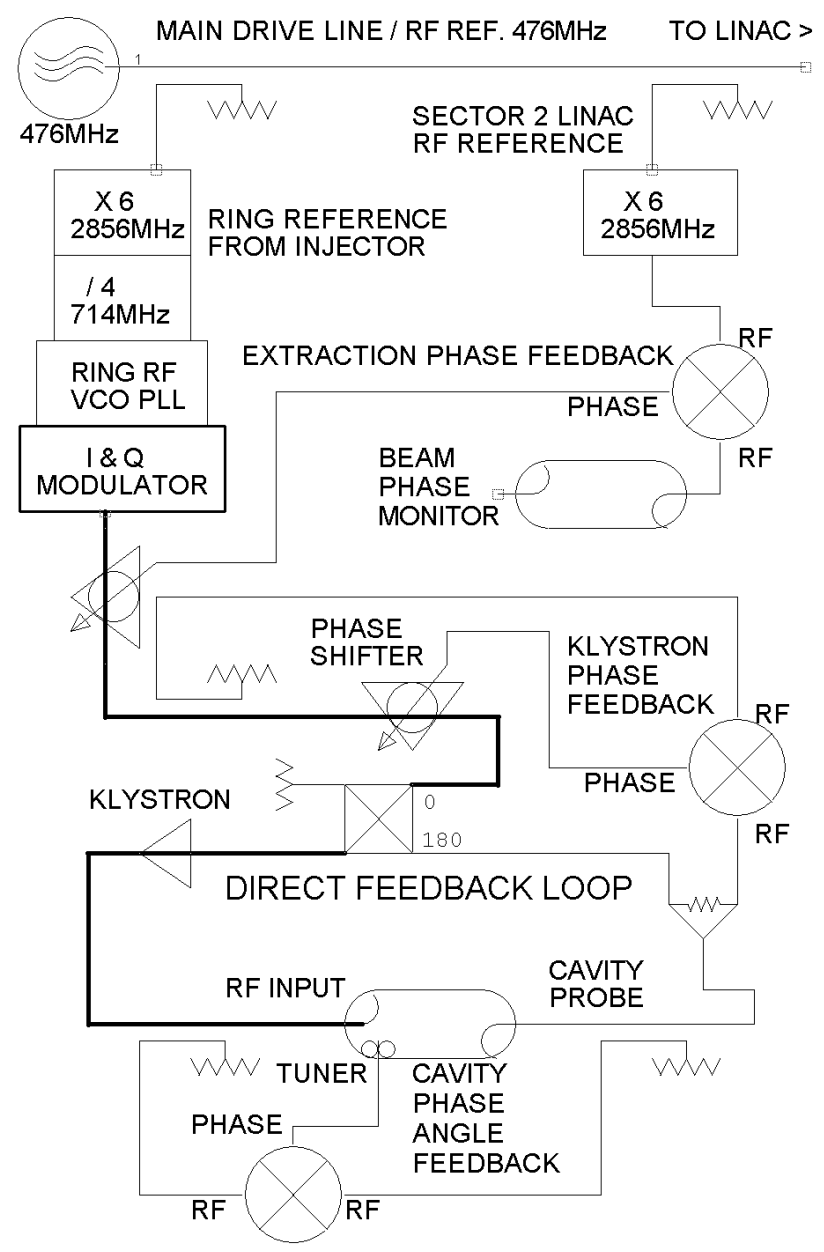

Figure 5. Damping Ring RF - Single Cavity Shown.

As the ring frequency returns to the original value the horizontal damping time and equilibrium emittance change back to their original values. It is desired therefore to stop the phase rotation and return to the original frequency as close to extraction as possible. To keep energy jitter below that which will effect luminosity, the ring extraction phase of the beam with respect to the linac must have an RMS value below 0.1 degree at 714.000MHz. The extraction feedback loop must be given enough time to stabilize from after locking and to before extraction. The extraction feedback loop was closed $200 \mu \mathrm{s}$ before extraction in order to minimize emittance and extraction jitter.

\section{OPERATION}

The system was installed and operated in the south damping ring initially. Files were set up to run the frequency shift at several different frequencies. Each of the frequencies allowed the modulator to run for a multiple of 84 rotations over $6.8 \mathrm{~ms}$. This requirement caused the frequency offset to be an integer multiple of $12.353 \mathrm{kHz}$. The frequency shift was started about $1.33 \mathrm{~ms}$ into the store. Initial damping of the injection transients and stabilization of the beam occurred during this first $1.33 \mathrm{~ms}$. Sampling of the RF for the cavity phase angle loop took place $1.0 \mathrm{mS}$ after injection. The frequency shift ended $200 \mu$ s before extraction.

The instantaneous frequency shift at the start and stop of the phase rotation caused a phase jump that kicked the beam. In order to minimize the effect the phase rotation was ramped up at the start and down at the end over a period of $200 \mu$ s, see Figure 6.

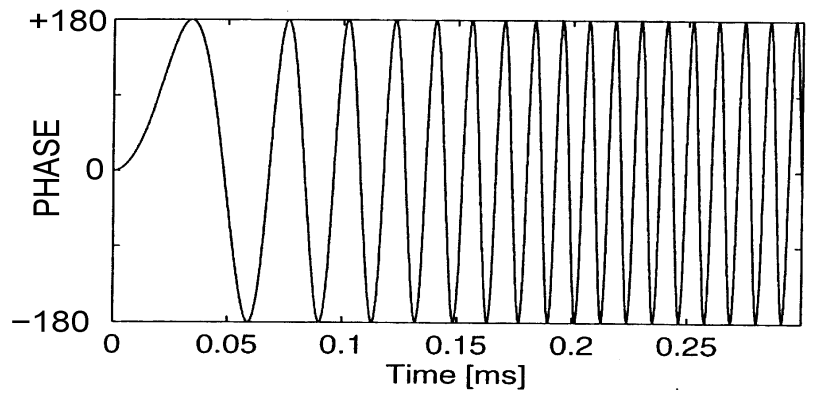

Figure 6. Ramp of Phase Rotation for $200 \mu \mathrm{s}$.

The tuning angle of one of the cavities was not able to be set to the desired value due to outgassing of a newly installed tuner. The cavities were unable to maintain the voltage during the frequency shift. In order not to saturate the voltage feedback loop, the voltage desired value was ramped to a lower value as the frequency was increased. The south damping ring has been run with the mid-store frequency shift and a reduction in emittance noted, Reference 1.

\section{ACKNOWLEDGEMENTS}

The authors would like to acknowledge Keith Jobe, Joe Frisch, Marc Ross, and Frank Zimmermann for their ideas, suggestions and encouragement, Doug McCormick for his assistance in tying the I \& Q modulator system into the damping ring control system, and R. Siemann.

\section{REFERENCES}

1. M.G. Minty et al., "Emittance Reduction via Dynamic RF Frequency Shift at the SLC Damping Rings," SLAC-PUB-7954, Stanford Linear Accelerator Center, Stanford, CA Sept. 1998; To be published in proc. of 17th Intl Conf on High-Energy Acc., Dubna, Russia (1998). 